\title{
Evidence based medicine: the Cochrane Collaboration
}

\begin{abstract}
What is the Cochrane Collaboration?
The Cochrane Collaboration is an international network of individuals and groups working in health care who are interested in developing and maintaining comprehensive, regularly updated, critical reviews of evidence from randomised controlled trials relevant to their specialty interests.
\end{abstract}

Systematic review aims at reducing the biases that in the past have been evident in some of the reviews submitted to or commissioned by journals and publishing houses. Perhaps the greatest virtue of systematic review is that its methods are transparent as well as rigorous, and are clearly laid out. Reviews can be readily updated when new evidence becomes available, and can be corrected in response to comment and criticism.

Systematic review includes a number of stages:

- Definition of the primary objective of the review

- Identification of all possible relevant trials by rigorous searching methods

- Assessment of the quality of each trial that meets the entry criteria for the review

- Extraction of data from each of these studies and contacting the authors if necessary for further information

- Data synthesis; this may be qualitative or quantitative

- Assessment of the implications for practice and further research.

Finding randomised trials is considerably more difficult than might be imagined. Existing databases (Medline, EMBASE) neither list all relevant journals nor reliably identify randomised trials, though this has improved in recent years. The Cochrane Collaboration has prepared searching strategies to make the best use of Medline, but even so the yield of randomised trials in some areas is less than half of the total number. Two additional strategies, therefore, have great importance. The first is hand searching of specialty journals. The second is identifying unpublished trials that may be in research theses held in universities, or may simply not have been published but be known to members of the sports injuries community. Often, in the past, such trials have not been published because they had negative results or lacked power. Therefore, there has been a potential publication bias towards trials with positive results.

In each specialty the work of individual reviewers is coordinated within a Cochrane Review Group (CRG). Many CRGs are fortunate to receive funding from government or charitable sources to maintain an editorial base where a full time researcher coordinates the group's activities in producing systematic reviews. CRGs have now been established in many areas of medicine. Although the Cochrane Centres provide support collaborative review groups are required to find funding for themselves. For individual members of groups, the main contribution is in time and intellectual effort.

The main products of the Cochrane Collaboration are the Cochrane Database of Systematic Reviews (CDSR) and the Cochrane Controlled Trials Register, which are published quarterly in electronic form in the Cochrane Library (Update Software, PO Box 696, Oxford OX2 7YX, UK).

\section{The Musculo-skeletal Injuries Group}

The Musculo-skeletal Injuries Group has already embarked on a number of reviews which should be relevant in the field of sports injury. The group aims at reviewing interventions tested by randomised controlled trials used in the prevention, management, and rehabilitation of fractures, dislocations, and soft tissue injuries of the musculoskeletal system.

Relevant reviews in preparation include :

Prevention:

- Prevention of ankle sprains

- Prevention of soft tissue injuries of the knee

- Prevention of lower limb stress fractures.

Treatment

- Treatment of acute and recurrent anterior shoulder dislocation

- Management of knee ligament and meniscal injuries

- Overuse patellofemoral pain

- Treatment of Achilles tendonitis

- Management of acute Achilles tendon ruptures

- Management of injuries to the lateral ligament complex of the ankle

- Treatment of lower limb stress fractures

- Heel pain/plantar fasciitis.

All these existing and potential activities need enthusiastic and committed participants to establish the process and keep it going. Completed reviews are, similarly, prepared in a standard format using the provided Review Management Software (REVMAN) and submitted to peer review before publication in the $C D S R$. The lead reviewer should make a commitment to maintain and update the review regularly.

A growing number of editors of journals have agreed that reviews lodged in the Cochrane Collaboration may also be submitted (appropriately formatted) for journal publication. Thus individual researchers continue to have the opportunity to publish in peer reviewed journals in addition to creating electronic publications in the Cochrane Database.

Individual members of a CRG can contribute by participating in an existing review, by initiating new reviews, or by carrying out hand searching of journals which might have relevant trials in their field of interest. This would be particularly relevant for smaller national surgical journals which may not be listed in Medline. The identification of any unpublished trials in the field of interest is also of value.

Interested individuals or groups should contact the Musculo-skeletal Injuries Group.

Professor WJ Gillespie
Professor of Orthopaedic
Surgery
Department of Orthopaedic
Surgery
Clinical Research Unit
Princess Margaret Rose
Orthopaedic Hospital
Fairmilehead
Edinburgh EH10 7ED
Telephone 0131-536-4667
Facsimile 0131-536-4754
Email
WJ.Gillespie@ed.ac.uk

Professor WJ Gillespie Surgery

Department of Orthopaedic Fairmilehead

Edinburgh EH10 7ED

Facsimile 0131-536-4754

WJ.Gillespie@ed.ac.uk
Dr Helen Handoll

Co-ordinator,

Musculo-skeletal Injuries Group

Department of Orthopaedic Surgery

Clinical Research Unit

Princess Margaret Rose Orthopaedic Hospital Fairmilehead

Edinburgh EH10 7ED

Telephone 0131-536-4669

Facsimile 0131-536-4845

Email H.Handoll@ ed.ac.uk 